\title{
Surgical Management of Ocular Hypotony
}

\author{
U. DEMELER \\ Bremen, West Germany
}

\begin{abstract}
Summary
Over the last 5 years, at the Eye Department in Bremen, 13 eyes with persistent hypotony have been treated by a refixation of the detached ciliary body, the so-called 'direct cyclopexy'. Ten eyes became hypotonous because of a traumatic cyclodialysis and 3 eyes as a result of an antiglaucomatous cyclodialysis surgery. The duration of the hypotony was from 10 days to 3 months, and the various cyclodialyses extended from 30 to 120 degrees. The postoperative follow-up was from 6 months to 5 years. The intraocular pressure was stabilised in all 10 eyes with traumatic cyclodialysis. In the 3 eyes with postoperative cyclodialysis all needed further antiglaucomatous procedures because of high intraocular pressure. In all 13 eyes the typical signs of hypotony disappeared postoperatively and visual improvement was achieved. The surgical procedure is discussed.
\end{abstract}

Persistent hypotony following surgery of the anterior segment is an uncommon but significant complication. Cyclodialysis is often complicated by hypotony, and occasionally goniotomy or trabeculotomy is followed by very low intraocular pressure in the absence of leakage from the surgical wound. Under these circumstances, the hypotony is usually associated with a detachment of the ciliary body, which may be secondary to a cyclodialysis cleft inadvertently created at the time of the surgery.

An excessive reduction in intraocular pressure without wound leakage can also occur after perforating injury or blunt ocular trauma.

In 1918, Collins ${ }^{1}$ undertook the pathological examination of rabbit eyes with persistent hypotony after trauma, and in every case there was a separation of the ciliary body up to the scleral spur. This produced a direct communication between the anterior chamber and the suprachoroidal space. The hypotony, associated with a cyclodialysis cleft was secondary to a decrease in production of aqueous as a result of detachment of the ciliary body and not from an increase in aqueous outflow.

The most important clinical features of persistent ocular hypotony are: Thickening of the cornea with striae of Descemet's membrane, shallow anterior chamber, choroidal detachment, disc oedema, tortuosity of the retinal vessels, thickening and striae of the retina including macular folds. All these factors lead to reduced visual acuity.

Many methods of eliminating excessive hypotony due to a traumatically or surgically induced cyclodialysis have been attempted in the past, all of which aim at closure of the cleft between the ciliary body and the scleral spur.

First attempts to fix the ciliary body to the sclera were undertaken by Vannas and Bjorkenheim. ${ }^{2}$ They did a limbal section with coagulation from the inside, in the region of the cleft. But within 10 days the intraocular pressure had risen to $70 \mathrm{mmHg}$. Maumenee and Stark $^{3}$ achieved the refixation of ciliary body to sclera by application of diathermy to the scleral lamella and in 1972 Hager $^{4}$ advised 
the insertion of bovine albumin between sclera and ciliary body. Techniques with similar principles have been described by Mackensen and Corydon, ${ }^{5}$ Naumann and Volcker $^{6}$ as well as Shea and Mednick. ${ }^{7}$

\section{Patients and Methods}

During the last 5 years we have performed a cycloplexy in 13 eyes with persistent ocular hypotony due to unintentional cyclodialysis or ocular trauma.

In 3 eyes an unintentional cyclodialyis occurred; after trabeculotomy in one case, and after goniotomies in the two others. Two eyes suffered a perforating injury and 8 eyes blunt trauma following accidents from a squash or tennis ball or a champagne cork, and all these cases resulted in ocular hypotony. In all eyes, the pre-operative intraocular pressure was below $4 \mathrm{mmHg}$.

The post-operative follow-up ranged from 6 months up to 5 years.

\section{Surgical Technique}

The procedure is done under general anaesthesia. In some eyes with a shallow anterior chamber, it can be very difficult to find the location and extent of the cyclodialysis. In these cases, the anterior chamber should be filled with a balanced salt solution to increase the intraocular pressure and deepen the anterior chamber. The angle can then be opened and the location and size of the cleft can be determined through a gonioscopic lens.

A limbus based conjunctival flap is fashioned over the extent of the cyclodialysis. A partial thickness scleral flap is then formed by making an incision 3 to $4 \mathrm{~mm}$ from the limbus and dissecting the flap towards the cornea. The deeper scleral lamella is incised 1 to $2 \mathrm{~mm}$ from the limbus and extended the full length of the scleral bed. On entering the supraciliary space, the diagnosis of ciliary body separation can be made, if clear fluid emerges through the incision or if the ciliary body is not found to be adherent to the sclera. Interrupted $10 / 0$ nylon sutures are inserted through a small piece of ciliary muscle and through the anterior lip of the sclera, at the scleral spur. Provided the interrupted sutures are very close together no further manipulations, like insertion of bovine albumin, application of cryo- or diathermy coagulation are necessary. No residual cleft or anterior synechiae remain after this procedure and normal chamber angle and normal intraocular pressure should result.

The deeper scleral lamella 1 to $2 \mathrm{~mm}$ from the limbus is sutured to the superior scleral lamella, the incision 3 to $4 \mathrm{~mm}$ from the limbus is closed with interrupted $10 / 0$ nylon sutures in order to achieve a double wound closure, this avoiding wound dehiscence. Finally the conjunctiva is closed with a running resorbable 8/0 Dexon suture.

\section{Results}

In 10 eyes with traumatic cyclodialysis, the post-operative intraocular pressure was between 11 and $18 \mathrm{mmHg}$ without further medical treatment. During the first post-operative weeks however, a secondary, postoperative rise in intraocular pressure occurred in some eyes, this was associated with inflammation and was easily controlled by topical timolol and osmotic agents.

We found, as expected, in the 3 glaucomatous eyes a postoperative intraocular pressure, ranging from $30-40 \mathrm{mmHg}$. After conservative treatment and cyclocryocoagulation failed to reduce the intraocular pressure, a partial excision of the ciliary body through the pars plicata succeeded in reducing the intraocular pressure to normal levels.

The duration of the ocular hypotony ranged from 10 days up to 3 months before the cyclopexy was performed. The duration of recovery, that is the resolution of the ocular hypotony, was surprisingly not related to the duration of the hypotony. In all 13 eyes, corneal thickening, choroidal detachment and macula folds disappeared within 3 to 10 days of the cyclopexy.

The same was true of the visual acuity, which was improved in 9 eyes. We would have expected a better visual recovery in those eyes, having had hypotony for only 10 days, but the same visual improvement was found in those eyes with a longer period of hypotony. It seems, that an eye can survive ocular hypotony over a long period of time without irreversible damage. In the 3 eyes whose post- 
operative visual acuity did not improve, choroidal rupture and macular scars were found to be responsible. In one eye with glaucoma the vision deteriorated because of further visual field loss caused by the high post-operative pressure.

\section{Conclusion}

In three patients with persistent hypotony following an inadvertent cyclodialysis procedure and in 10 patients after a severe ocular trauma to the eye, direct refixation of the detached ciliary body to the scleral spur was effective in restoring the intraocular pressure, in eliminating the clinical consequences of hypotony in the anterior and posterior segments and in improving visual acuity. The technical procedure is very simple, the detached ciliary muscle is refixed to the scleral spur with $10 / 0$ nylon sutures placed very close to each other under a partial thickness scleral flap which is sutured separately in order to achieve a double wound closure. No other manipulations like cryo- or diathermycoagulation or bovine albumin are necessary to close the cyclodialysis cleit.

\section{References}

'Collins ET: An experimental investigation as to some of the effects of hypotony in rabbits eyes. Trans Ophthalmol Soc UK 1918, 38: 217-27.

2 Vannas M and Bjordengeim B: On Hypotony following Cyclodialysis and its Treatment. Acta Opgthalmol (Kbh) 1952, 30: 63-4.

${ }^{3}$ Maumenee A, Edward Stark, Walter J: Management of persistent Hypotony after planned or inadvertent Cyclodialysis. Am J Ophthalmol 1971, 71: 10-16.

${ }^{4}$ Hager H: Besondere mikrochirurgische Eingriffe. Klin Mbl Augenheilk 1972, 161: 265-72.

${ }^{5}$ Makensen G and Corydon L: Verbesserter Eingriff gegen das Hypertonie-Syndrom mit Kammerwinkespalt nach drucksenkender Operation. Klin Mbl Augenheilk 1974, 165: 696-704.

${ }^{6}$ Naumann GOH and Volcker HE: Direkte Zylopexie zur Behandlung des persistierenden Hypotonie-Syndroms infolge traumatischer Zyklodialyse. Klin Mbl Augenheilk 1981, 179: 266-70.

${ }^{7}$ Shea M and Mednick, Edward B: Ciliary body Reattachment in Ocular Hypotony. Arch Ophthalmol 1981, 99: 279-81. 